\title{
PAPR reduction in OFDM system with Wavelet transformation and partial transmit sequence technique
}

\author{
Nurina Febryanti ${ }^{1^{*}}$, Dadang Gunawan ${ }^{2}$ \\ 1, 2 Department of Electrical Engineering, University of Indonesia, Depok, Indonesia
}

\author{
Index Terms \\ OFDM \\ PAPR \\ Wavelet-OFDM \\ PTS \\ Received: 26 July 2016 \\ Accepted: 28 August 2016 \\ Published: 12 February 2017
}

\begin{abstract}
Orthogonal Frequency Division Multiplexing (OFDM) system is a multicarrier system used to transfer data at high rate. One of the drawbacks of OFDM is the high value of Peak to Average Power Ratio (PAPR). High PAPR values will mitigate the efficacy of the power amplifier. This paper discusses the Wavelet-OFDM with Partial Transmit Sequence. The use of wavelet transformation on the OFDM system provides several advantages compared to Fourier transform. These advantages include high efficiency due to the loss of guard interval (GI). The proposed Wavelet-PTS system reduces the PAPR significantly, which can achieve a reduction of $8 \mathrm{~dB}$ PAPR compared with the conventional OFDM system.
\end{abstract}

(C) 2017 The Author(s). Published by TAF Publishing.

\section{INTRODUCTION}

Wireless communication is expected to provide high speed data services in available bandwidth with good performance. OFDM is also prominent method for high-speed communications. OFDM is a transmission technique that uses multiple orthogonal pieces of frequency (multicarrier). One of the disadvantages of OFDM systems is the high Peak-to Average Power Ratio (PAPR). High PAPR causes inefficiency of the power amplifier. Therefore, PAPR should be reduced. Some PAPR reduction methods have been proposed, such as clipping and filtering, SLM, etc. Generally, the reduction methods can be divided into three categories: distortion method, such as clipping and filtering; distortion less or probabilistic method, such as Partial Transmit Sequence (PTS) and Selective Mapping; and coding method such as Golay complementary sequences [1]. The simplest PAPR reduction technique and easiest to implement in OFDM systems is Clipping and Filtering [2], but the technique decreases the bit error rate (BER). In [3], the PTS and
SLM techniques in OFDM systems were compared. PTS and SLM techniques reduce the PAPR by reducing the probability of appearance of high PAPR value. The simulation results in [3] showed that the PTS technique achieved better PAPR mitigation when comparing with the SLM method in OFDM system. All the PAPR reduction methods described in [1] deal with Discrete Fourier Transform (DFT)-based OFDM. Another variant of OFDM, called discrete wavelet transform (DWT)-based OFDM, was studied in [4-6]. Wavelet based OFDM is a best substitute to Fast Fourier Transform-based Orthogonal Frequency Division Multiplexing (FFT-OFDM). W-OFDM has several advantages such as more resistant to Inter-symbol Interference (ISI) and Inter-Carrier Interference (ICI) as well as high efficiency due to the loss of Guard Interval (GI) [4] and [9]. In OWDM, GI can be eliminated because of the nature of its inter-sub-band mutually orthogonal. Moreover, the use of DWT in place of DFT can also reduce PAPR. In W-OFDM, wavelets preferred over sinusoids to utilize to sustain sub-carrier. In this paper we propose a PAPR mitigation technique form on Wavelet transformation and Partial Transmit Sequence scheme (WAV-PTS).

\footnotetext{
${ }^{*}$ Corresponding author: Nurina Febryant

${ }^{\dagger}$ Email: nurina.febryanti@ui.ac.id
} 


\section{WAVELET TRANSFORMATION}

\section{A. Wavelet-Based OFDM}

Fig. 1 shows a picture of the most basic discrete wavelet transformation process and only performing 1 (one) time signal decomposition.

Discrete wavelet transformation of an input $\mathrm{x}$ signal can be calculated by passing the input signal into a row filter. In other words, the results of discrete transformation of an input $x$ signal are obtained from a process of decomposition. The decomposition process signal here is the process of convolution of the input $\mathrm{x}$ signal with a low pass filter which has the impulse $g$ response and by the presence of convolution process of the input $x$ signal with a high pass filter which has impulse $h$ response. The process of decomposition of the input $x$ signal with a low pass filter will produce approximation coefficient while the process of decomposition of the input $x$ signal with a high pass filter will produce detail coefficients. So that the discrete wavelet transformation process to the input $\mathrm{x}$ signal, will produce 2 (two) outputs, that are Approximation coefficients and Detail coefficients.

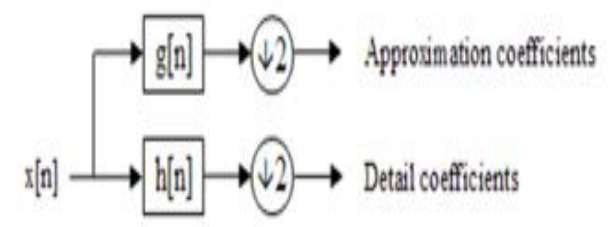

Fig. 1. Block diagram of Wavelet decomposition

where

$\mathrm{x}[\mathrm{n}]$ : The original signal

$\mathrm{g}[\mathrm{n}]$ : Low-Pass Filter (LPF)

$\mathrm{h}[\mathrm{n}]$ : High-Pass Filter (HPF)

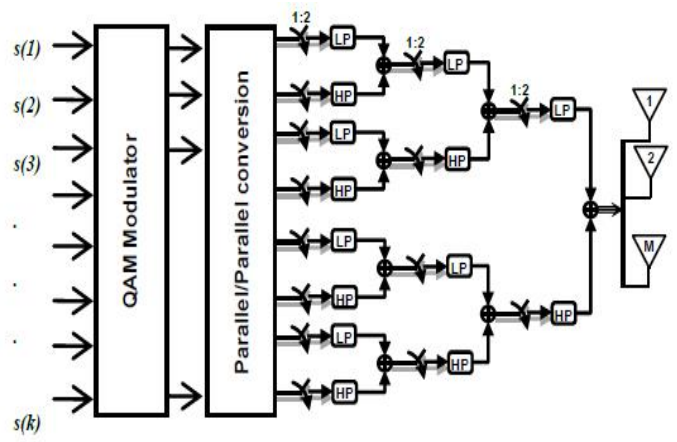

Fig. 2. W-OFDM Transmitter [7]
Fig. 2 shows the transmitter configurations. On the transmitter side, first a quadrature amplitude modulation is used for mapping $\mathrm{s}(\mathrm{k})$ data stream to the symbol stream $\mathrm{x}(\mathrm{n})$. After the mapping process a parallel-to-parallel (P/P) converter reshapes the modulated data stream $\mathrm{x}(\mathrm{n})$ into; for example, $\mathrm{N}=8$ parallel data streams. This $\mathrm{P} / \mathrm{P}$ converter makes sure that $N=2 n$ where $n$ is an integer, so that the transmitter can perform Inverse Discrete Wavelet Transformation (IDWT) and produce one final sequence in "n" stages.

Sequential two $\mathrm{x}(\mathrm{n})$ symbol streams are up-sampled by the up-sampling factor 2 , filtered by the wavelet filter $\mathrm{g}(\mathrm{n})$ or $\mathrm{h}(\mathrm{n})$, respectively, and then summed. Output sequences are up-sampled by 2 , filtered and summed again. The up-sampling and filtering processes continue until one single output stream is obtained.

\section{B. Wavelet Families}

In general, there are two categories of wavelet families: orthogonal and biorthogonal, where the orthogonality of the wavelet family is related to the filter coefficients. Orthogonal wavelet family includes wavelet Daubechies (Db), wavelet Symlet (sym), and wavelet Coiflet (coif). Biorthogonal wavelet family includes BiorSplines (bior) and ReverseBior (rbio). Orthogonal wavelets are parameterized by filter order $(\mathrm{N})$ that determines filter length (L). Biorthogonal wavelets use filters with similar or dissimilar orders for decomposition $(\mathrm{Nd})$ and reconstruction $(\mathrm{Nr})$. Although the filter length is determined by the filter order $\mathrm{N}$, the relationship between them is different in different wavelet families.

\section{CONVENTIONAL PARTIAL TRANSMIT SEQUENCE}

PTS is one of the widespread techniques used to reduce PAPR in OFDM system which is implemented in this paper. The basic philosophy of this method is to subdividing OFDM symbol data into sub-data which are transmitted and by sub-blocks and multiplied by weighing value.

The block diagram for PTS technique implementation is shown in Figure 2. The data sequence $\mathrm{X}$ in frequency domain is sub-divided into $\mathrm{v}$ sub-sequences which are transmitted in sub-blocks without overlapping and having equal size of $\mathrm{N}$ which contains $\mathrm{N} / \mathrm{V}$ non-zero values in each sub-block.

With the assumption that the sub-blocks have equal size without having any gap between them. Thus the subblock vector in frequency domain can be written as follows: 


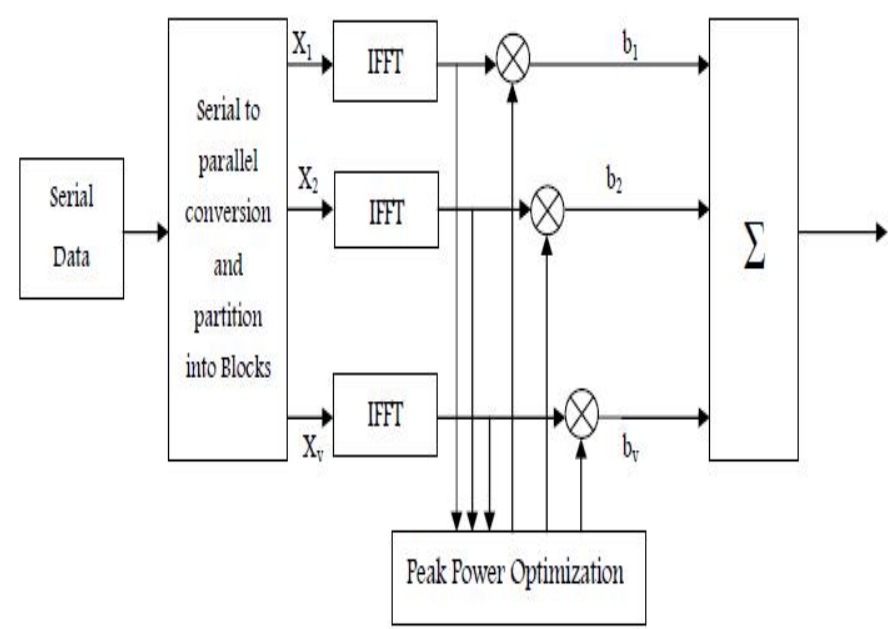

Fig. 3. Block diagram of conventional partial transmit sequence

$$
X=\sum_{v=1}^{v} b_{v} X_{v}
$$

Where $b^{(v)}=e^{j \varphi v}\left(\varphi_{v} \varepsilon[0,2 \pi]\right) v=1,2,3, \cdots, X_{v}$ is the weighing factor which is used for phase rotation. The time domain for the above sub-block vector can be yielded by applying IFFT function, that is

$x=\operatorname{IFFT}(X)=\sum_{v=1}^{v} b_{v} X_{v}$

Based on the equations above the peak value optimization is performed by selecting the parameter $b_{v}$, so that the value of PAPR can be minimized.

\section{PROPOSED WAVELET-PTS SCHEME}

In this method, Inverse Fourier Transform was replaced by Inverse Discrete Wavelet Transform. First, the data were divided into sub-blocks, and then the information signal in the form of bits will be converted into a parallel through several channels and sub-channels. This signal was then mapped by using QPSK modulator into a complex symbol. The output of the QPSK modulator would be an input to the IDWT block to be processed based on wavelet transform. The process in IDWT block itself is a substitute of IFFT process for conventional OFDM systems.

W-OFDM signal (Wavelet-Orthogonal Frequency Division Multiplexing) comes out after passing through the IDWT block leading to the Partial Transmit Sequence (PTS) block, which aims to further reduce the PAPR, where the output of IDWT multiplied by a weighting factor to achieve the lowest PAPR. The block image of WAV-PTS structure is presented in Figure 5. The aim is optimal combination of sub-blocks in order to get to get time domain OFDM signals.

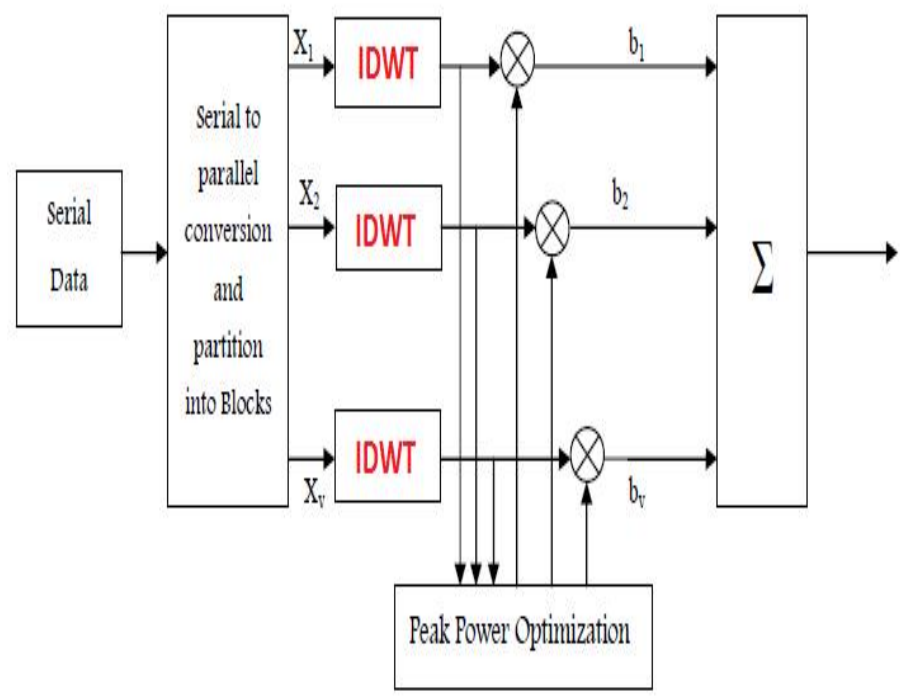

Fig. 4. Block image Wavelet-PTS structure

\section{SIMULATED FINDINGS OF EXPERIMEN}

\section{A. Determination of Wavelet Type Used}

First simulation using Wavelet Transformation with different types of wavelet families to get the most optimal wavelet in reducing PAPR for use in a combination WaveletPTS system. The wavelet families used in this paper are summarized in Table.1.

TABLE 1

WAVELET FAMILIES USED

\begin{tabular}{ll}
\hline \hline Wavelet Families & Wavelet Orders \\
\hline Daubechies (db) & $\begin{array}{l}\mathrm{db} 7, \mathrm{db}, \mathrm{db} 9, \mathrm{db} 10, \\
\mathrm{db} 11, \mathrm{db} 12, \mathrm{db} 13\end{array}$ \\
Symlet (sym) & sym7, sym8, sym9, s- \\
& ym10, sym11, sym12, sym13 \\
Coiflet (coif) & coif2, coif3, coif4, coif5 \\
BiorSplines (bior)- & rbio2.6, rbio3.5, rbio3.9, \\
ReverseBior (rbio) &, rbio5.5, rbio6.8, bior5.5, bior6.8 \\
\hline \hline
\end{tabular}

To analyze PAPR distribution, a statistical parameter called Complementary Cumulative Distribution Function (CCDF) is usually used. CCDF gives a probability that PAPR exceeds a certain value. The CCDF results for the W-OFDM systems using wavelet families compared with the DFTOFDM are shown in Fig. 6 - Fig. 9 . 


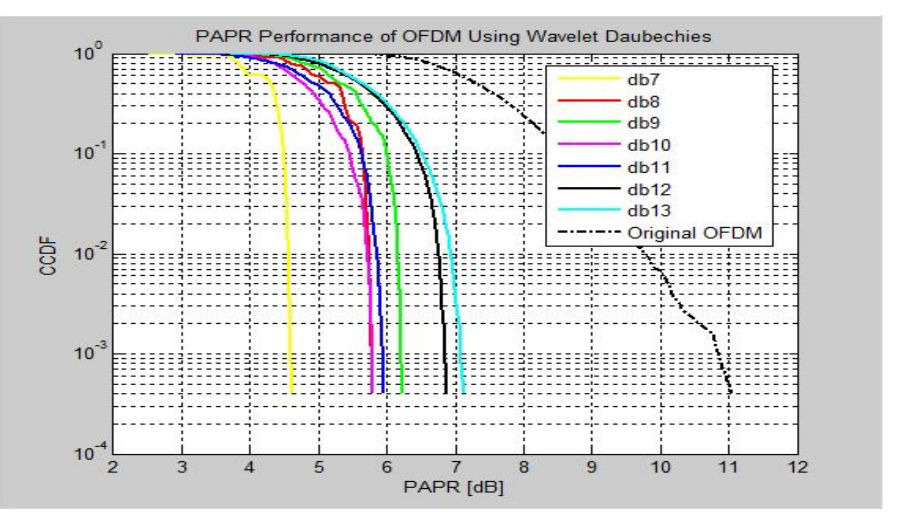

Fig. 5 . PAPR comparative analysis of various Daubechies family presented through CCDF vs PAPR curve

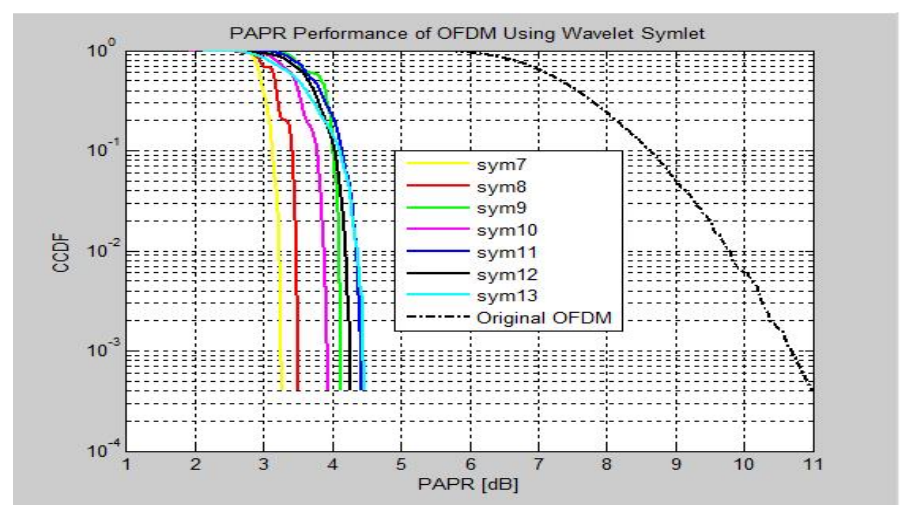

Fig. 6. PAPR comparative analysis of various Symlet families presented through CCDF vs PAPR curve

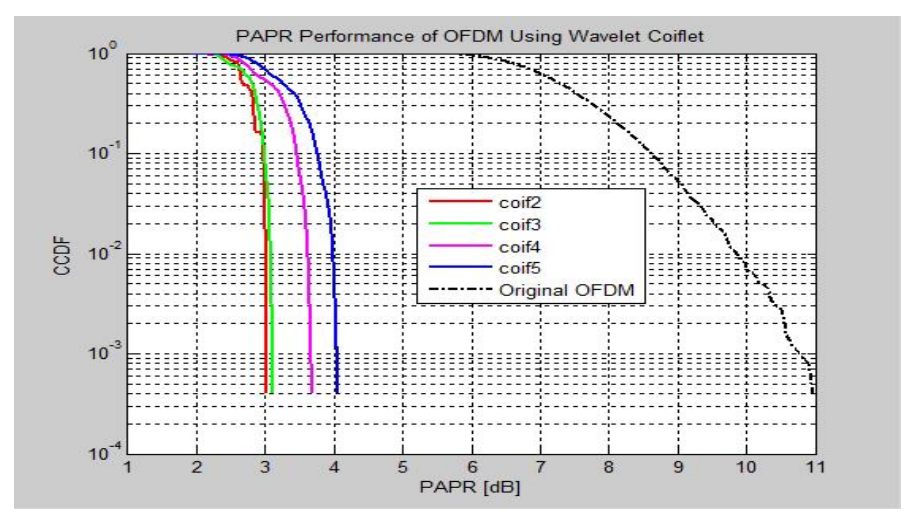

Fig. 7 . PAPR comparative analysis of various Coiflet families presented through CCDF vs PAPR curve

We can observe from Fig. 6 that W-OFDM using $\mathrm{db} 7$ has the lowest PAPR. The difference is about 6.8 $\mathrm{dB}$ compared with the conventional OFDM at probability of 10-3. In Fig. 7 the lowest PAPR distribution in W-OFDM is achieved by using sym7, which is about $7.7 \mathrm{~dB}$ lower than conventional OFDM. In Fig. 8 we can observe that W-
OFDM using coif2 has the lowest PAPR compared with conventional OFDM which is about $8.4 \mathrm{~dB}$. Fig. 9 shows the CCDF for biorthogonal wavelet families compared with conventional OFDM. The PAPR distributions of W-OFDM using biorthogonal wavelet functions have only little difference. However, we can observe that W-OFDM using rbio3.5 has the lowest PAPR which is about $7.5 \mathrm{~dB}$.

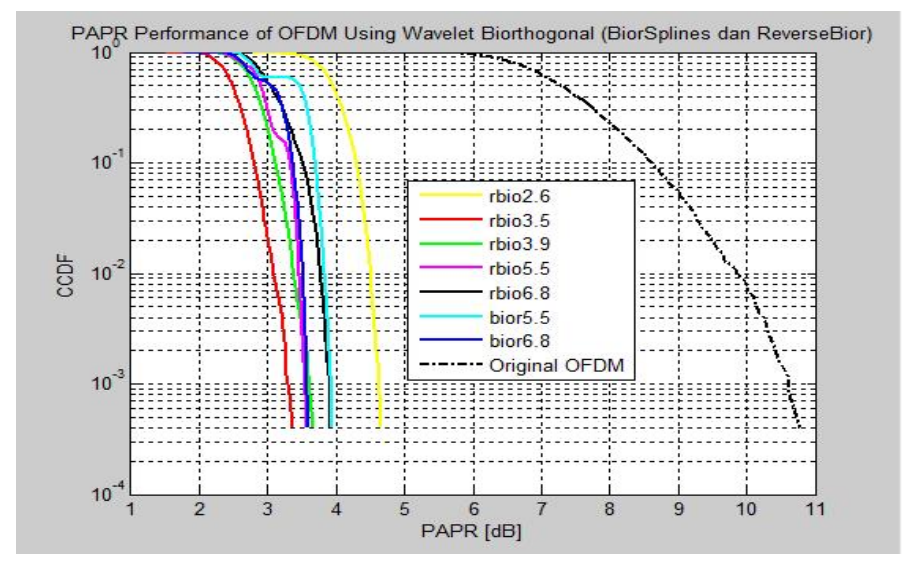

Fig. 8. PAPR comparative analysis of various Biorthogonal families presented through CCDF vs PAPR curve

Output OWDM system provided several advantages compared with OFDM. W-OFDM system generated PAPR value which was much smaller than OFDM. The use of different wavelet families will give different output PAPR. In orthogonal wavelet, wavelet with a small order is more advisable to be implemented, because with the small order, performance PAPR that being resulting from will also decrease. While on biorthogonal wavelet, the increase in PAPR reduction does not depend on the increase in the order of N. It may be caused by a combination of the selection order $\mathrm{Nr}$ and $\mathrm{Nd}$ conducted for the most excellent PAPR reduction.

The results comparison showed that the wavelet Coiflet_2 (coif2) is a type of best wavelet to reduce PAPR in OFDM system with a reduction value up to $8.4 \mathrm{~dB}$. Therefore, wavelet Coiflet_2 (coif2) is used for the analysis of OFDM system that uses a combination of wavelet transform and Partial Transmit Sequence techniques in the next section.

\section{B. Wavelet-PTS Simulation}

In present study, the proposed new OFDM multicarrier scheme is based on a combination of Wavelet Transform and PTS. The comparative analysis of PAPR is between 
the suggested Wavelet-PTS, conventional PTS, W-OFDM and the signal of original OFDM originally generated. The findings are presented in figure 9.

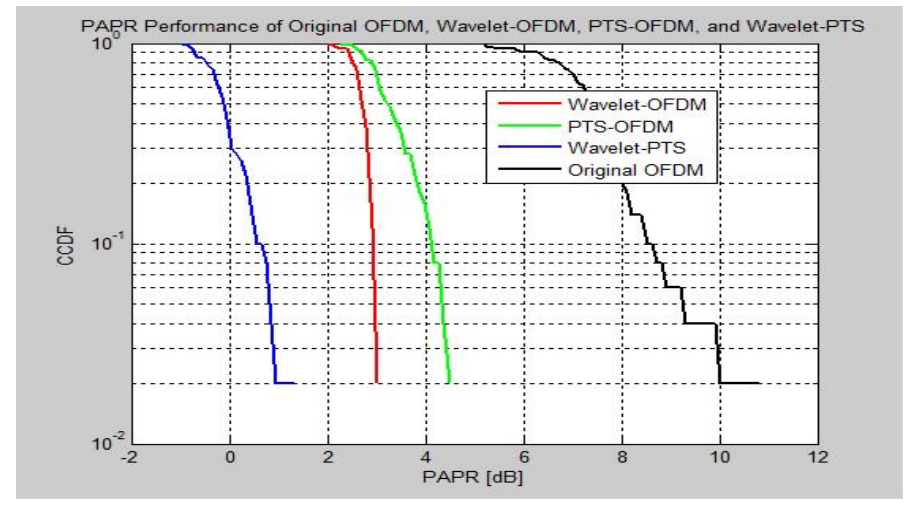

Fig. 9. CCDF vs PAPR curve represents comparative analysis Wavelet-PTS, conventional PTS, W-OFDM and the original signal

The Wavelet-PTS shows the excellent PAPR mitigation of $0.95 \mathrm{~dB}$ with comparison to conventional PTS $4 \mathrm{~dB}$ at probability of $10^{-1}$, W-OFDM $3 \mathrm{~dB}$ and original OFDM signal $9 \mathrm{~dB}$. It has been noted that suggested scheme Wavelet-PTS mitigates the PAPR up to $8 \mathrm{~dB}$ as compared to original OFDM signal.

\section{CONCLUSION}

The use of techniques of PTS can increase system performance Wavelet-OFDM (W-OFDM) in reducing PAPR. The proposed scheme shows better performance than the Wavelet scheme and conventional PTS in terms of PAPR reduction. Wavelet-PTS that was proposed achieved PAPR reduction in amount of $8 \mathrm{~dB}$ better than Conventional OFDM scheme.

So, the use a combination of schemes Wavelet and Partial Transmit Sequence scheme that was proposed can significantly reduce PAPR reduction when compared with Wavelet-OFDM scheme or Conventional PTS. The combination of wavelet transforms and PTS techniques can reduce optimally PAPR in OFDM systems.

\section{REFERENCES}

[1] N. Sharma, "Peak-to-average power ratio reduction techniques for OFDM signals," International Journal of Computer Applications, vol. 96, no. 22, pp. 40-45, 2012.

[2] S. P. Yadav and S. C. Bera, "PAPR reduction using Clipping and filtering technique for nonlinear communication systems," in International Conference on Computing, Communication and Automation (ICCCA), Greater Noida, India, 2015. DOI: 10.1109/CCAA.2015.7148590

[3] S. S. Bhadoriya and M. R. Bhatia, "Comparison of PTS and SLM technique in OFDM System," International Journal of Engineering Sciences Management (IJESM), vol. 5, no. 1, pp. 7-10, 2012.

[4] N. Kumar, "BER analysis of conventional and wavelet based OFDM in LTE using different modulation techniques," in International Conference on Recent Advances in Engineering and Computational Sciences (RAECS), Chandigarh, India, 2014.

[5] T. Pargtee, S. Mitra and S. C. Bhawani, "BER analysis of conventional OFDM (DFT-OFDM) and Wavelet-based OFDM (DWT-OFDM)," International Journal on Recent and Innovation Trends in Computing and Communication, vol. 3, no. 1, pp. 423-426, 2015. DOI: 10.17762/ijritcc2321-8169.150184

[6] A. Delphina Josline and N. Hariprasad, "Studies on DWT based OFDM for LTE system," ARPN Journal of Engineering and Applied Sciences vol. 10, no. 9, pp. 3901-3904, 2015.

[7] M. M. Hasan and S. S. Singh, "PAPR analysis of FFT and Wavelet based OFDM systems for wireless communications," International Journal of Computer Applications, vol. 60, no. 15, pp. 38-42, 2012.

[8] R. Gayathri, V. Sangeetha, S. Prabha, D. Meenakshi and N. R. Raajan, "PAPR reduction in OFDM using partial transmit Sequence (PTS)," International Journal of Engineering and Technology (IJET), vol. 5 no. 2, pp. 1428-1431, 2013.

[9] M. M. Reza and M. H. U. Ahmed "A traffic transmission scheme for smart grid communication," International Journal of Technology and Engineering Studies, vol. 2, no. 6, pp. 164-171, 2016. DoI: 10.20469/ijtes.2.40001-6 